\title{
Computational Analysis for Roughness-Based Transition Control
}

Fei Li, Meelan M. Choudhari, Chau-Lyan Chang and Jack R. Edwards

\begin{abstract}
Suitably placed discrete roughness elements are known to delay or hasten the onset of transition, depending on requirements. In this paper, 2D eigenvalue analysis is used to study the effects of surface roughness in the context of transition delay over subsonic and supersonic swept wing configurations, as well as boundarylayer tripping on the forebody of a hypersonic air breathing vehicle.
\end{abstract}

\section{Introduction}

Surface roughness is known to have a substantial impact on the aerodynamic or aerothermodynamic predictions for a flight vehicle, regardless of the state of the boundary layer. When the incoming boundary-layer flow is laminar, the presence of 3D surface roughness tends to accelerate the laminar-turbulent transition process, although carefully placed spanwise periodic discrete roughness elements can delay the onset of transition in crossflow dominated boundary-layer flows. Passive control via roughness elements provides an attractive avenue for drag reduction on subsonic and supersonic swept wing configurations. On the other hand, in scramjet applications, artificial roughness is often employed for tripping the boundary layer flow on the forebody of the vehicle to prevent engine unstart and to minimize the flow non-

Fei Li

NASA Langley Reseacrh Center, Hampton, VA 23681 e-mail: fei.li@nasa.gov

Meelan M. Choudhari

NASA Langley Reseacrh Center, Hampton, VA 23681 e-mail: meelan.m.choudhari@nasa.gov

Chau-Lyan Chang

NASA Langley Research Center, Hampton, VA 23681 e-mail: chau-lyan.chang@nasa.gov

Jack R. Edwards

North Carolina State University, Raleigh, NC 27693 e-mail: jredward@ncsu.edu 
uniformities at the entrance to the combustor inlet.

This paper uses spatial, 2D eigenvalue analysis to explore the physics of roughness effects on boundary layer transition across subsonic through hypersonic regimes via a study of selected flow configurations.

\section{Methods}

The equations governing the unstable small-amplitude perturbations are obtained by linearizing the Navier-Stokes equations about a specified basic state, such as a finite amplitude crossflow vortex developing in a swept-airfoil boundary layer or longitudinal streaks behind a spanwise-periodic array of boundary layer trips. For a basic state that is slowly varying along one of the spatial coordinates (e.g. the crossflow vortex axis), using a wave ansatz in that direction reduces the spatial dimension of the problem governing the disturbance quantities by one, resulting in a set of two-dimensional, linear partial differential equations at the leading order. The 2-D eigenvalue problem in a spatial framework is solved to characterize the amplification characteristics of high-frequency, secondary instabilities of crossflow vortices as well as the instabilities of the stationary streaks trailing a periodic array of roughness elements. The spatial stability equations are formulated in a non-orthogonal coordinate system in order to properly account for the direction of spatial growth and the spanwise periodicity of the unstable perturbations (see $\mathrm{Li}$ and Choudhari 2008).

\section{Results}

Computations are carried out to study the effects of surface roughness in the context of three specific configurations, viz., (1) transition delay over a subsonic swept wing at chord Reynolds number of $R e_{c}=7 \times 10^{6}$ (Carpenter et al. 2008); (2) transition control over a swept wing at Mach 2.4 and $R e_{c}=16 \times 10^{6}$ (Saric and Reed 2002) and (3) boundary layer tripping over a scaled model of the Hyper-X forebody at Mach 6 (Berry et al. 2001). For cases (1) and (2), the amplitude that is often referred to below is that of the velocity component in the direction of the normal chord as a fraction of the freestream velocity, and for case (3) it refers to the velocity component in the direction of the freestream.

In case (1), the effect of periodically spaced roughness elements is simulated by introducing stationary crossflow vortices with a spanwise wavelength of $2.25 \mathrm{~mm}$ (the control mode) to suppress the growth of the linearly more unstable stationary crossflow mode with a spanwise wavelength of $4.5 \mathrm{~mm}$ (the target mode). Computations based on nonlinear Parabolized Stability Equations (PSE) have shown that 
the amplitudes of the target mode can be significantly reduced via a control mode with sufficiently large initial amplitudes. With the initial amplitude of the $4.5 \mathrm{~mm}$ primary stationary crossflow vortex mode fixed at $10^{-4}$, the effect of increasing the amplitude of the $2.25 \mathrm{~mm}$ control mode is shown in Figure 1 (a). With zero control amplitude, it is seen that both of the dominant $Y$ - and $Z$-mode secondary instabilities undergo strong modal amplification. As the control amplitude increases, modal amplification of both the dominant $Y$ - and $Z$-modes is weakened. Furthermore, as shown in Figure 1 (a), the presence of the control mode apparently affects the $Z$ mode of secondary instability much more strongly than the $Y$-mode. This indicates that, if indeed transition is caused by the growth of the $Z$-mode as some experiments seem to suggest (Kawakami, M. et al. 1999, White and Saric 2005), the DRE is a very effective means to achieve transition delay in the present case.

There is, however, a possibility that the $2.25 \mathrm{~mm}$ control mode could become susceptible to secondary instability and may itself break down to cause an earlier onset of transition (i.e. overcontrol). Secondary instability analysis for the $2.25 \mathrm{~mm}$ control mode is carried out to assess the likelihood of this scenario. The peak $\mathrm{N}$-factors for $Z$-mode of secondary instability are less than 1 for all three control inputs. Of the three initial amplitudes of $0.002,0.005$ and 0.01 , only the last gives rise to a maximum $Y$-mode secondary instability $\mathrm{N}$-factor of approximately 11 at a chordwise location close to $x / c=0.25$. For the other two amplitudes of control input, the $Y$-mode $\mathrm{N}$-factors remain less than or equal to approximately 8 . Therefore, even if the $Y$-mode can also lead to transition, the possibility of premature transition due to overcontrol can be avoided by keeping the initial control mode amplitudes below a
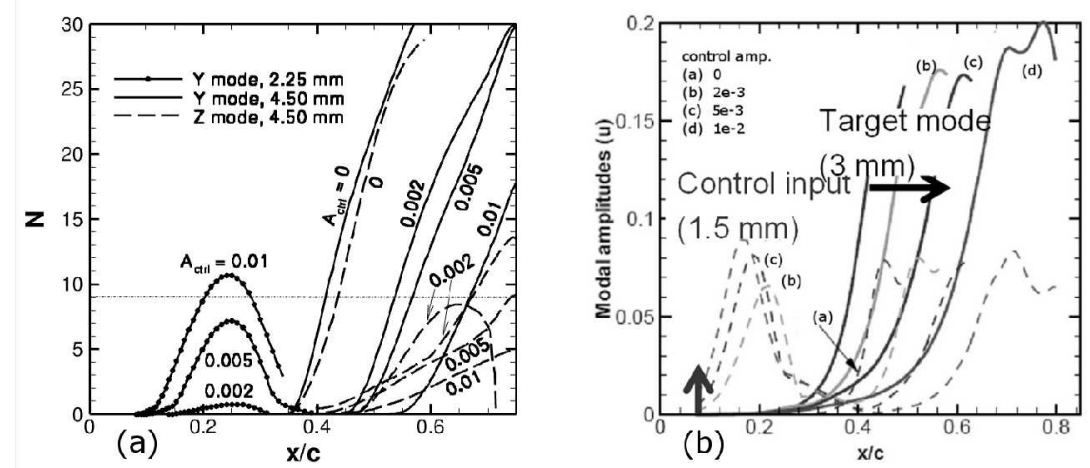

Fig. 1

(a) Subsonic swept wing configuration.Envelops of secondary N-factor curves for $Y$ - and $Z$ familes. Nonlinear control of most unstable mode $(4.5 \mathrm{~mm})$ via control input $(2.25 \mathrm{~mm})$ with different initial amplitudes.

(b) Mach 2.4 supersonic swept wing configuration. Crossflow vortex amplitude curves. Nonlinear control of most unstable mode $(3 \mathrm{~mm})$ via control input $(1.5 \mathrm{~mm})$ with different amplitudes 
certain threshold, which in this case appears to be around 0.005 or slightly larger.

In case (2), similar analyses as those in case (1) are carried out for a swept wing at a Mach number of 2.4. The wavelength of the control mode is $1.5 \mathrm{~mm}$, and that of the target mode is $3.0 \mathrm{~mm}$. The rapid amplitude rise of the $3 \mathrm{~mm}$ target mode is progressively delayed with increasing initial amplitude of the $1.5 \mathrm{~mm}$ control mode (Fig. 1 (b)). Computations of secondary instability confirm the accompanying delay in the amplification of the secondary instability modes on the $3 \mathrm{~mm}$ crossflow vortex. More interestingly, however, the secondary instability analysis on the $1.5 \mathrm{~mm}$ control mode reveals the increased likelihood of premature transiton via overcontrol, since the $\mathrm{N}$-factors of both the $Y$ - and the $Z$-modes of secondary instability reach relatively large values (with the $Y$-mode continuing to be the most dominant one). For an initial control amplitude of 0.001 , maximum $\mathrm{N}$-factors for the two modes are approxiamtely 8 and 6 , respectively. When the amplitude is increased to 0.002 , the peak $\mathrm{N}$-factors approach 13 and 9 , respectively.

In case (3), the roughness array is employed to trigger an earlier transition in a hypersonic boundary layer instead of delaying it. The computations described herein model the Hyper-X Mach 6 flow configuration with three flat ramps to provide the necessary compression ahead of the scramjet engine (see Berry et al. 2001). The trip elements produce strong trailing streaks (Fig. 2), which are susceptible to instabilities similar to those riding on finite amplitude Görtler vortices over a concave surface. The roughness array is placed on ramp 1 at 7.4 inches from the leading edge, and the two corners joining the three ramps are at 12.4 and 17.7 inches, respectively. The results presented herein correspond to a trip spacing of $\delta=0.081$ " and a peak height of $h=0.060$ ". The mean flow is computed with a Navier-Stokes solver, using the immersed boundary technique (see Ghosh et al. 2008).

Figure 2 shows that the streamwise streaks with strong spanwise boundary-layer displacement in the wake of the trip array would persisit for long distances over the

Fig. 2 Steamwise streaks produced behind the trip array on Hyper-X model (visualized via u-velocity contours at $x=6 ", 8 ", 10 ", 12$ " and 14 ", respectively, flow is from bottom left to top right and, for visual clarity, the wall normal and spanwise coordinates $\mathrm{Y}$ and $\mathrm{Z}$ have been modified relative to $\mathrm{X}$ coordinate). The underlying light surface corresponds to the isosurface of streamwise velocity for $u=1 \mathrm{~m} / \mathrm{s}$.

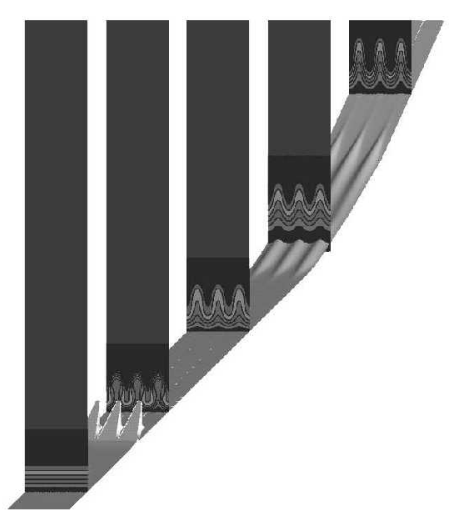


forebody surface if the boundary-layer flow were to remain laminar.

Since the spanwise and wall-normal length scales of these streaks are comparable with each other, the modified boundary-layer flow has a strongly inhomogeneous character in both $Y$ and $Z$ directions. Therefore, its stability characteristics are more appropriately studied by solving a $2 \mathrm{D}$ eigenvalue problem. It is found that multiple modes of instability co-exist, which is typical of boundary-layer flows modified by finite amplitude streaks. The growth rates of the two dominant modes of instability at $x=7.9$ " have been plotted against the disturbance frequency in Fig. 3(a), which also indicates the representative mode shapes for the magnitude of the $u$-velocity perturbation associated with each mode. The spanwise period of these modes is equal to the array spacing $\delta$. Despite the presence of strongly inflexional boundarylayer profiles in the wall-normal direction, the more unstable mode (i.e., mode 1) from abovementioned modes is found to be driven by the spanwise $(Z)$ shear of the basic state (i.e., corresponds to an odd mode, which induces sinuous motions of the underlying stationary streaks). Since the spanwise shear occurs solely because of the trip array, this dominant, odd (or $Z$ ) mode of streak instability would not have existed without the roughness elements. The subdominant mode 2 is found to be an even $(Y)$ mode.

$\mathrm{N}$-factors and growth rates of fixed frequency disturbances belonging to mode 1 family have been plotted in Figs. 3(b) and 3(c), respectively. As seen from Fig. 3(c), the growth of streak instability ceases well upstream of the end of the model ramp 1 $(x=12.4$ ") before resuming again over the second compression ramp. The absence of growth in the immediate vicinity of the compression corner is attributed to the rapid decrease in streak amplitudes just ahead of the corner. As seen from Fig. 3(b), the odd mode (mode 1) disturbances near $\mathrm{f}=90 \mathrm{kHz}$ reach an $\mathrm{N}$-factor of approximately 7 across an amplification region of just 2.5 inches. In a previous set of experiments in the same facility (Horvath et al. 2002), transition onset on a smooth, flared cone model had been found to correlate with $N \approx 4$. Thus, if a similar value of $\mathrm{N}$ is
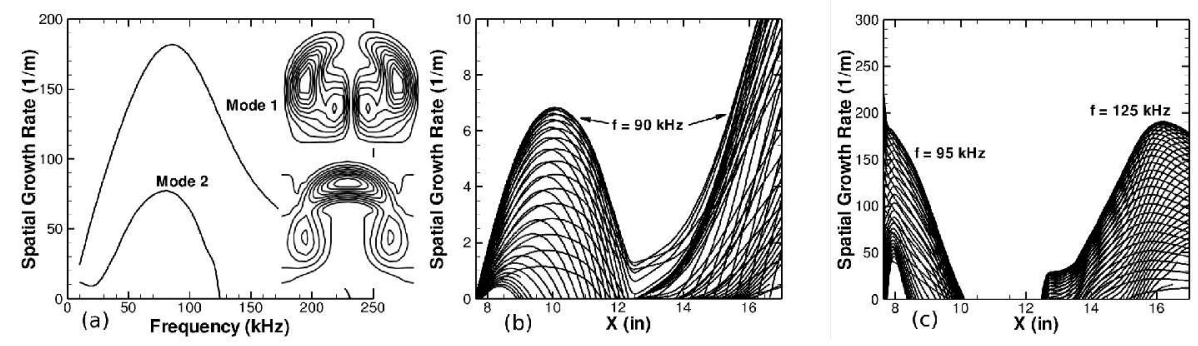

Fig. 3 For steamwise streaks produced behind the trip array on Hyper-X model.

(a) Growth rates and mode shapes of modes 1 and 2 at plane $x=7.9$ ".

(b) Mode $1 \mathrm{~N}$-factors.

(c) Mode 1 growth rate. 
assumed to correlate with the onset of roughness-induced transition on the Hyper-X model, then the predicted onset of transition will be within a distance of $\Delta x / L=$ 0.05 behind the trip (where $\mathrm{L}=48$ " denotes the reference length of the vehicle at the model scale). The phosphor thermography measurement in the experiment (Berry et al. 2001) is also suggestive of transition onset at a short distance behind the trip array.

\section{Conclusions}

By applying artificial roughness elements, transition can be either delayed or hastened, depending on the specific requirements. In the former case, carefully designed roughness elements produce long streamwise structures that suppress the growth of the more dangerous crossflow vortices and hence weaken the high frequency secondary instabilities that would otherwise cause transition to occur earlier. The 2D eigenvalue analysis plays an important role in this analysis by delineating an optimal range of control input magnitudes. In the case of tripping over compression surfaces, roughness elements generate stationary streaks that amplify across the compression corner and, furthermore, enhance the growth of nonstationary streak instabilities that are expected to trigger an earlier onset of transition as observed in the experiments.

\section{References}

1. Berry, S.A., Auslender, A.H., Dilley, A.D. and Calleja, J.F., "Hypersonic Boundary-Layer Trip Development for Hyper-X," J. Spacecraft and Rockets, vol. 38, No. 6, pp. 853-864, Nov.-Dec. 2001.

2. Carpenter, A.L. Saric, W.S., and Reed, H.L., "Laminar Flow Control on A Swept Wing With Distributed Roughnes," AIAA Paper 2008-7335, 2008.

3. Ghosh, S., Choi, J.-I., and Edwards, J.R. "RANS and hybrid LES/RANS Simulation of the Effects of Micro Vortex Generators using Immersed Boundary Methods," AIAA Paper 20083728, June, 2008

4. Kawakami, M., Kohama, Y. and Okutsu, M. "Stability Characteristics of Stationary Crossflow vortices," AIAA Paper 99-0811, 1999.

5. White E. B., and Saric W. S., "Secondary Instabilty of Crossflow Vortices," J. Fluid Mech. Vol. 525, pp. 275-308, 2005.

6. Li, F., and Choudhari, M., "Spatially Developing Secondary Instabilities and Attachment Line Instability in Supersonic Boundary Layers," AIAA Paper 2008-590, 2008.

7. Saric, W.S. and Reed, H.L., "Supersonic Laminar Flow Control on Swept wings Using Distributed Roughness." AIAA paper 2002-147, 2002. 